\section{MS15-O4 Molecular rotors in nanoporous periodic architectures}

Angiolina Comotti ${ }^{1}$, Bracco Silvia ${ }^{1}$, Donata Asnaghi ${ }^{1}$, Piero Sozzani ${ }^{1}$

1. University of Milano Bicocca, Department of Materials Science, Via R. Cozzi 55, 20125 Milano, Italy

email: angiolina.comotti@mater.unimib.it

New mesoporous hybrid covalent frameworks were prepared to realize a periodic architecture of fast molecular rotors containing dynamic C-F dipoles in their structure.[1] The mobile elements, designed on the basis of fluorinated p-divinylbenzene moieties, were integrated into the robust covalent structure through siloxane bonds, and showed not only the rapid dynamics of the aromatic rings (ca. $10^{8} \mathrm{~Hz}$ at $325 \mathrm{~K}$ ), as detected by solid-state NMR spectroscopy, but also a dielectric response typical of a fast dipole reorientation under the stimuli of an applied electric field. The nanochannels are open and accessible to diffusing-in gas molecules, and rotor mobility could be individually regulated by $\mathrm{I}_{\text {, vapors. The }}$ iodine enters the channels of the periodic architecture and selectively reacts with the pivotal double bonds of the divinyl-fluoro-phenylene rotors without disrupting the structure, affecting their motion and the dielectric properties.The combination of porosity with rotor dynamics was also discovered in molecular crystals.

Disulfonated rotor-containing molecular rods were self-assembled with alkylammonium salts to fabricate porous supramolecular architectures held together by charge-assisted hydrogen bonds (Figure 1) [2]. The rotors, as fast as $10^{8} \mathrm{~Hz}$ at $240 \mathrm{~K}$, are exposed to the crystalline channels, which absorb $\mathrm{CO}_{2}$ and $\mathrm{I}_{2}$ vapors at low pressure. The rotor dynamics could be switched off and on by $\mathrm{I}_{2}$ absorption/desorption, showing remarkable change of material dynamics by the interaction with gaseous species and suggesting the use of molecular crystals in sensing and pollutant management.

Moreover, porosity can be switched on/off in molecular crystals based on star-shaped azobenzene tetramers by photoirradiation[3]. Photoinduced trans-cis isomerization of molecules changes intermolecular interactions triggerring the formation of a non porous material which can be reverted to porous crystals by visible light irradiation or thermal treatment.

Ref.: 1) Angew. Chemie Int. Ed. 2015, ASAP (VIP Article); 2) J. Am. Chem. Soc. 2014, 136, 618; 3) Nature Chem. 2015, accepted.

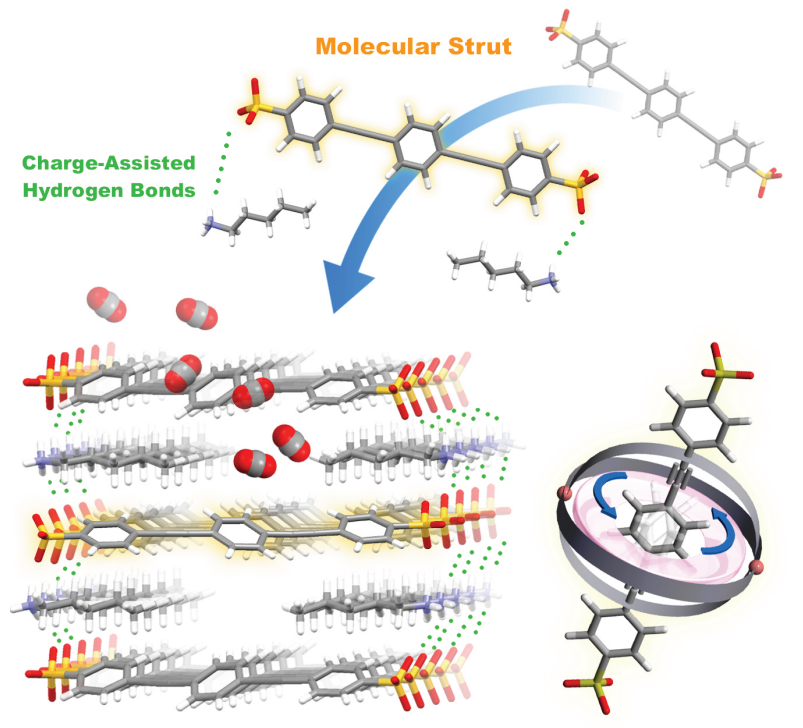

Molecular Rotors in Organic Porous Architecture

Figure 1.

Keywords: Porous Materials, Molecular Rotors 